\title{
A 3D wideband package solution using MCM-D BCB technology for tile TR module
}

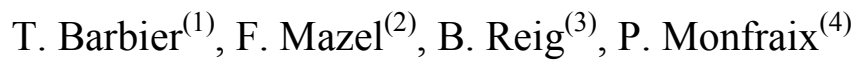 \\ (1) Thales Airborne Systems, - 2, avenue Gay-Lussac 78851 Elancourt - France \\ ${ }^{(2)}$ Thales Microwave, - 29, avenue Carnot 91349 Massy cedex - France \\ (3) Thales Research and Technologies, - Domaine de Corbeville 91404 Orsay cedex - France \\ ${ }^{(4)}$ Alcatel Space Industries, - 26, avenue J.F.Champollion 31037 Toulouse - France
}

\begin{abstract}
This paper describes the works performed on a solution of packaging dedicated to $3 D$ TR modules in the $2-20 \mathrm{GHz}$ frequency range. The proposed solution is based on a non hermetic architecture involving organic and BCB substrates. The studied key bricks relative to assembly, vertical interconnections, passives integration are described and results consecutive to electrical and environmental evaluation are presented. A 3D TR module demonstrator taking benefit from the key bricks evaluated through test vehicles has been realised and its performances measured when submitted to airborne environmental constraints.
\end{abstract}

\section{INTRODUCTION}

Tile antennas application is a significant improvement for the performances of TR modules for phased array antennas. An important benefit is the reduction in size and weight. TR modules which could present such characteristics can take place along the wings of an aircraft or an UAV, and also on the fuselage. The challenge is to propose a wide band package dedicated to airborne applications which encloses TR module functionalities operating over a large frequency spectrum: communication systems, radar, electronic warfare. For this, the packaging solution has to answer to specific requirements such as the $\mathrm{RF}$ and DC routing in the $3^{\text {rd }}$ dimension, a highly integration depending directly on the maximum operating frequency, and in consequence the thermal conditioning.

This paper presents the architecture of a 3D TR module, the evaluation of the main identified key bricks which led to realise a TR module demonstrator. Electrical results recorded during the environmental evaluation of this demonstrator conclude the paper.

\section{3D TR MODULE ARCHITECTURE}

The 3D TR module is inserted between radiating elements and control circuits which ensure both the RF and DC signals distribution.

Considering one TR module per radiating element, the area of the TR module is strongly dependant of the mesh of the radiating panel. In this application, the radiating panel operates at $18 \mathrm{GHz}$. Correct performances of the phased array antenna at this frequency supposes a $9,5 \mathrm{~mm}$ spacing between elements. This leads to propose a packaging solution where four TR modules are grouped in the same package, called quadpack, which optimize the MMIC integration area. Fig. 1 presents a front view of the general construction of the 3D quadpack.

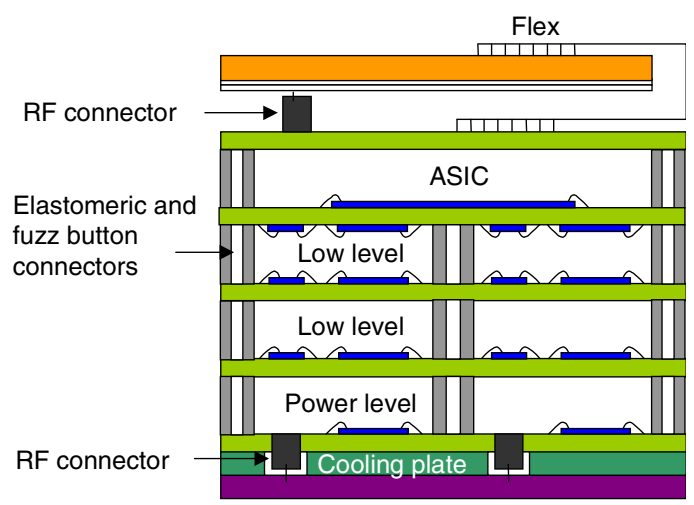

Fig. 1. MCM-D architecture

The quadpack is assembled onto a cooling plate which realises the mechanical interface between the TR modules and the antenna. The power levels of the quadpack are placed close to the cooling plate in order to optimize the thermal management. Such an architecture reduces also the number of transitions between the high power MMICs of the transmit channel and the antenna. On the upper stages are placed the low power levels. RF and DC signals transit through solderless vertical connectors enclosed inside metallic rings which separate the different layers and ensure isolation and shielding between adjacent TR modules. On the top of the quadpack, an ASIC, common to the four channels controls the attenuators and phase shifters placed on the layers just below. The layers are made up of organic substrates (Ro4003®) except for the high power level. The size of the involved MMIC (High Power Amplifier, Intermediate Amplifier) and the associated bias circuit require a highly integration, brought by multi-layers thin films technology, using BCB dielectric (MCM-D BCB technology).

\section{KEY BRICKS INVESTIGATION}

Some technological bricks have been identified to be evaluated in order to be integrated in the future demonstrator based on the architecture described here above. The main challenges are:

1) The vertical RF interconnections between levels made up of Ro4003 $\AA$ based layers (low power level) or BCB multilayers (high power levels), 
2) The external RF interconnections involving subminiature connectors and flex between the quadpack, the antenna and the control circuit,

3) The DC vertical interconnection between the levels,

4) The BCB multilayer in which will be integrated embedded bias capacitors,

5) The shielding between TR modules in the same package

6) The evaluation under severe environment of the assembly.

\section{A. RF interconnection between levels}

The vertical RF interconnections between the RF levels are performed using solderless $1,1 \mathrm{~mm}$ diameter Fuzz buttons connectors developed by TECKNIT. These connectors are enclosed into the metallic rings which ensure the ground continuity. Compression is about $80 \mathrm{~g}$ per contact which is compliant with an assembly using conductive glue between layers and metallic ring. Some tests vehicles have been realised to evaluate the $2-20 \mathrm{GHz}$ transitions between two Ro4003 ${ }^{\circledR}$ based layers and also between BCB and Ro4003 ${ }^{\circledR}$ layers. Return losses for one transition between Ro4003 $\AA$ based layers is better than $-15 \mathrm{~dB}$ with insertion losses which do not exceed $0,8 \mathrm{~dB}$ at $20 \mathrm{GHz}$ (see Fig. 2). Concerning the transitions using Fuzz-buttons between BCB and Ro4003® based layers, return losses are better than $-10 \mathrm{~dB}$ for 2 transitions and insertion losses, less than $-1,8 \mathrm{~dB}$ at $20 \mathrm{GHz}$ (see Fig. 3).

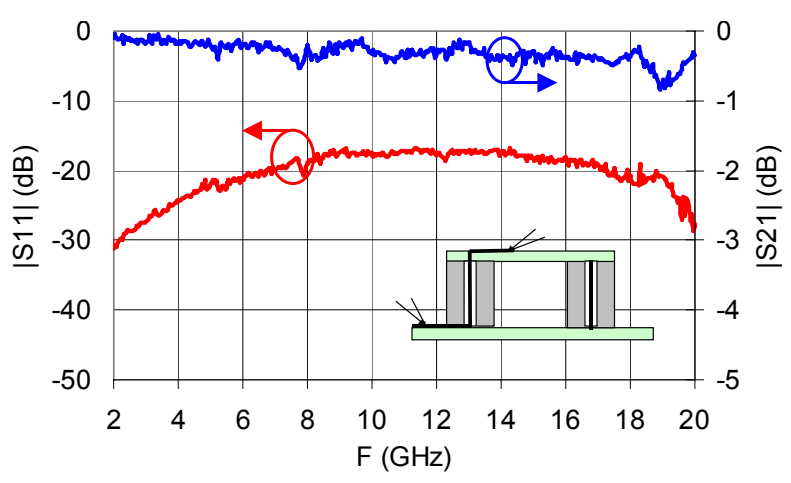

Fig. 2. Fuzz-button transition between Ro4003® substrates

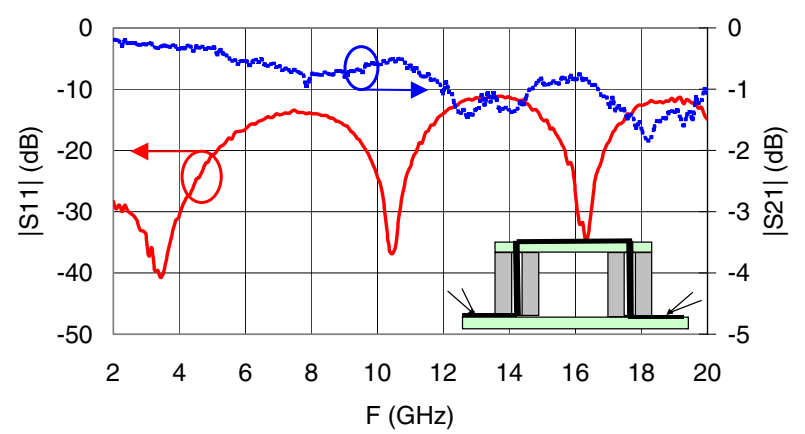

Fig. 3. Fuzz-button transitions between Ro4003 ${ }^{\circledR}$ and BCB substrates

\section{B. RF external interconnection}

Two solutions have been investigated to realise the external interconnections: GPPO connector from GILBERT inserted into the cooling plate and realizing the interconnection between the TR modules of the quadpack and the antenna, and a flex solution for the interconnection between quadpack and control circuit. Transition between BCB substrate and GPPO connectors gave not satisfaction mainly due to the dimensions of the connector which are not compliant with the pipes inside the cooling plate. A Fuzz button solution is more appropriate for this external interconnection.

The 3D coplanar interconnection by flex has been

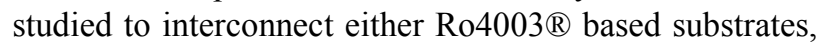
or $\mathrm{BCB}$ based substrates. In each case, the substrates are stacked and separated by a distance of 2,5 mm. A RF flex made up of a $50 \mu \mathrm{m}$ thick Kapton and $17 \mu \mathrm{m}$ thick plated copper has been simulated and realised. Fig. 4 shows the flex before and after assembly on a test vehicle involving two BCB based layers. The return losses measured on the RF accesses of each $\mathrm{BCB}$ based layers are better than $-12 \mathrm{~dB}$ over the $2-20 \mathrm{GHz}$ frequency range.
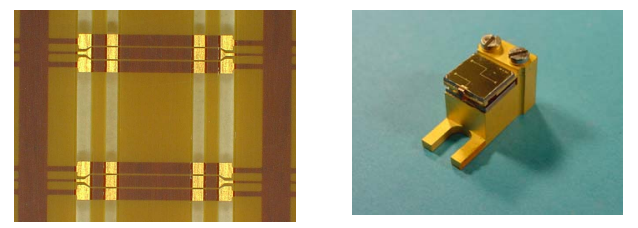

Fig. 4. 3D coplanar flex, before and after assembly on its test vehicle

\section{DC vertical interconnection}

The DC vertical interconnection is performed with an elastomeric connector made up of two rows of $50 \mu \mathrm{m}$ diameter wires with a $100 \mu \mathrm{m}$ spacing enclosed in a $7 \mathrm{~mm}$ length dielectric. These connectors are also enclosed in the metallic rings. A test vehicle composed of three layers interconnected by these connectors has been realised. DC pads on the layers are $400 \times 400 \mu \mathrm{m}^{2}$. The electric resistance is less than $0.1 \Omega$ for each connector with a current capacity higher than $3 \mathrm{~A}$. The test vehicle is measured after 500 thermal cycling $\left(-55^{\circ} \mathrm{C}\right.$ to $\left.+125^{\circ} \mathrm{C}\right)$. The height of the test vehicles made up of three layers separated by two metallic rings increased by only $10 \mu \mathrm{m}$ after 500 thermal cycling.

\section{MCM-D BCB evaluation}

One benefit of the MCM-D BCB technology is the possibility to integrate Ta2O5 capacitors inside the $\mathrm{BCB}$ substrate (see Fig. 5). The density of the capacitance is $300 \mathrm{pF} / \mathrm{mm}^{2}$. Embedded capacitances from $45 \mathrm{pF}$ to $550 \mathrm{pF}$ have been evaluated in adequate test vehicles. Measurements showed an accuracy of the capacitance value which is $+/-10 \%$. No degradation (open or short circuits) have been recorded after 500 thermal cycling endured by the test vehicles. 
The electrical characterisation of the $\mathrm{BCB}$ substrate shows $0,9 \mathrm{~dB} / \mathrm{cm}$ insertion loss at $20 \mathrm{GHz}$ for a $18 \mu \mathrm{m}$ thick substrate, and $0,4 \mathrm{~dB} / \mathrm{cm}$ insertion loss for a $45 \mu \mathrm{m}$ thick substrate. The electrical resistance of the vias varies from $5 \mathrm{~m} \Omega$ to $6 \mathrm{~m} \Omega$ whatever the thickness of the substrate.

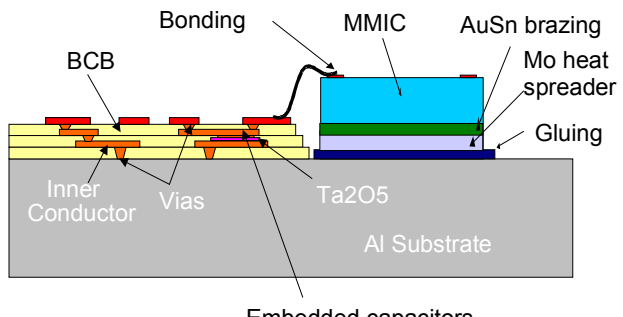

Fig. 5. Ta2O5 embedded capacitors in BCB substrate

\section{E. Shielding between TR modules}

A test vehicle has been realised to measure the shielding between TR modules of the same quadpack. Measurements have been performed to measure the coupling between RF lines on each side of a plated area on which is glued the metallic ring. Fig. 6 illustrates the test vehicle. Measurements before the assembly of the metallic ring showed $40 \mathrm{~dB}$ coupling in the worst case (distance between the lines, number of ground vias on the plated area). Measurements performed after the assembly of the metallic ring on the plated area showed a shielding between cavities better than $55 \mathrm{~dB}$ up to $20 \mathrm{GHz}$.

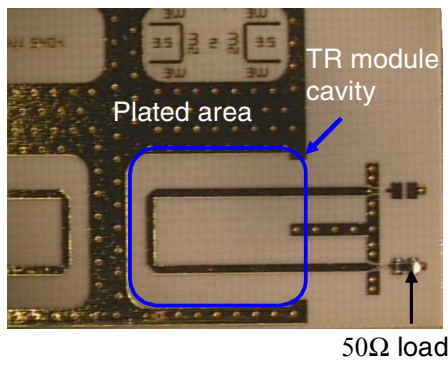

Fig. 6. Shielding test vehicle

\section{F. Environmental evaluation of the assemblies}

Some test vehicles involving a low noise amplifier assembled on a Ro4003 ${ }^{\circledR}$ based layer, and a high power amplifier connected on a BCB based multilayer have been realised, measured and submitted to severe environment: 500 thermal cycling between $-55^{\circ} \mathrm{C}$ and $+125^{\circ} \mathrm{C}$, life test at $125^{\circ} \mathrm{C}$ for $1000 \mathrm{~h}$, salt spray $\left(35^{\circ} \mathrm{C}\right.$, $5 \%$ ) during $48 \mathrm{~h}$. Intermediate electrical measurements (Sparameters, noise figure, output power) have been performed to record the behaviour evolution. The return losses evolution does not exceed $2 \mathrm{~dB}$ both for LNA and HPA test vehicles after thermal cycling, life test and salt spray. The gain evolution is $1 \mathrm{~dB}$ maximum for the two types of test vehicles after the whole program.

\section{DEMONSTRATOR MEASUREMENTS}

A demonstrator has then be realised taking benefit from the key bricks evaluation. The block diagram is made up of a transmit channel (Triquint TGA9092 HPA associated with a UMS foundry intermediate driver and bias circuits), and a receive channel made up of two $13 \mathrm{~dB}$ gain LNAs (UMS foundry). A low level RF switch, integrated on the low level stage enables to collect the RF signal from receive channel or send the same signal to the transmit channel. Fig. 7 illustrates the MCM-D BCB demonstrator. The quadpack is realised to integrate four TR modules, even if only one TR module among the four ones is integrated in every demonstrator. The three remaining cavities have been employed to route DC and $\mathrm{RF}$ lines in order to test each equipped layer before $3 \mathrm{D}$ assembly. The interconnections between cavities on the same layer were then removed as illustrated on Fig. 8. The external RF interconnection towards antenna is not implemented due to the fact that the cooling plate has not been studied in the program. RF and DC signals are provided from the top of the quadpack through probes to the TR module.

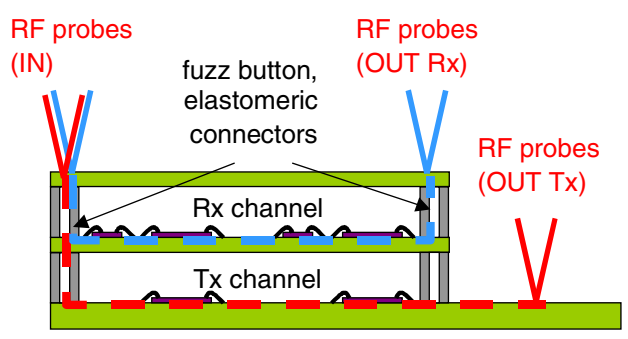

Fig. 7. MCM-D BCB demonstrator signal access

Seven layers dedicated to the transmit channel are realised, tested and assembled with seven low level layers, also tested separately. Fig. 8 shows one of the demonstrators once assembled.

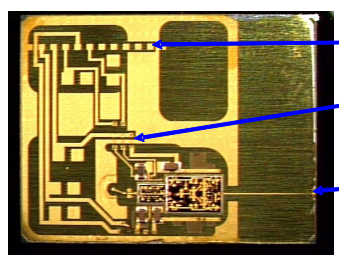

DC probes Access Removal bondings RF transmit channel output

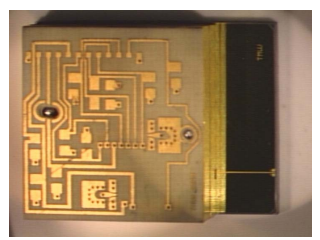

Fig. 8. High power level before and after 3D assembly

Measurements have been performed on five demonstrators. Performances are grouped between demonstrators. Transmit gain is better than $35 \mathrm{~dB}$ in the $6-18 \mathrm{GHz}$ frequency range, output power is $+32 \mathrm{dBm}$ average for $-5 \mathrm{dBm}$ input $\mathrm{RF}$ level. Concerning the receive channel, gain is better than $20 \mathrm{~dB}$ and noise figure less than $6 \mathrm{~dB}$ in the $4-18 \mathrm{GHz}$ frequency range. 
The demonstrators have been then evaluated under environmental constraints: 500 thermal cycling $\left(-55^{\circ} \mathrm{C}\right.$, $+125^{\circ} \mathrm{C}$ ), unbiased HAST $\left(110^{\circ} \mathrm{C}, 85 \%\right)$ for $264 \mathrm{~h}$, vibrations and mechanical shocks up to $500 \mathrm{~g}$. Except transmit RF channel access on BCB which damaged after repetitive probes positioning, the demonstrators did not recorded important performance deviations after the environmental program (gain deviation less than $1 \mathrm{~dB}$ on transmit and receive channels).

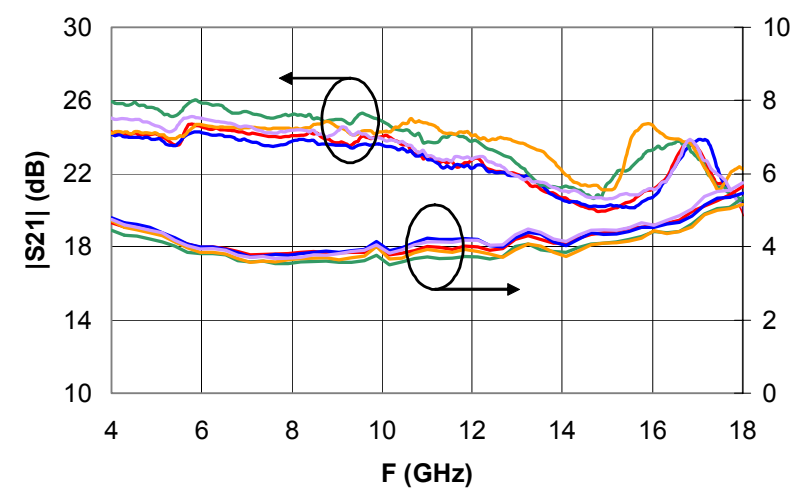

Fig. 9. Receive gain and noise figure recorded on 5 demonstrators

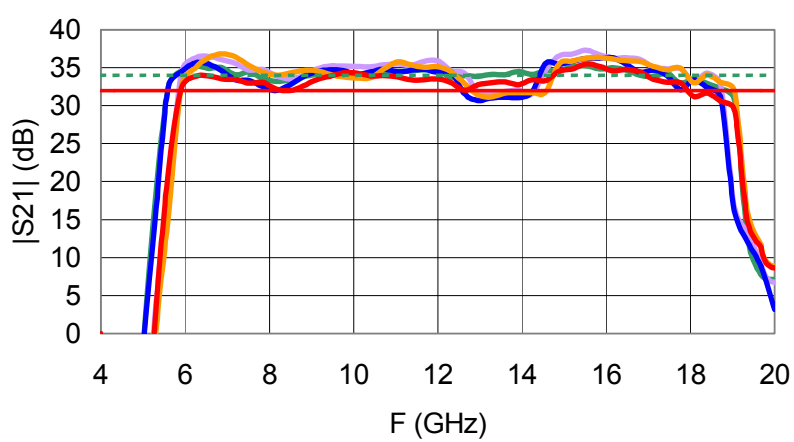

Fig. 10. Transmit gain recorded on 5 demonstrators

\section{CONCLUSION}

A 3D architecture for tile wideband TR module was designed. The high level of integration of this technology contributes to reduce the module size and to be compliant with a tile architecture antenna. Technological key bricks of this architecture have been developed and encouraging results have been obtained. A demonstrator using these key bricks has been realised and its performance evaluated under environmental constraints. The good results obtained on this non hermetic solution, especially after unbiased HAST enable to propose this assembly for airborne tile antennas.

\section{ACKNOWLEDGEMENT}

The authors wish to acknowledge the French Administration DGA and CELAR which supported the program.

( $\quad$ Registered Trademark Rogers Corporation. 